\title{
Arc Suppression Coatings for Electrodynamic Tethers and Spacecraft Cabling
}

\author{
Sven G. Bilén* \\ The Pennsylvania State University, University Park, PA 16802 \\ and \\ Brian E. Gilchrist ${ }^{\dagger}$ \\ The University of Michigan, Ann Arbor, MI 48109
}

\begin{abstract}
Advanced insulation materials are needed to support future electrodynamic tether missions as well as for other high voltage applications requiring direct exposure to vacuum/space plasma conditions, such as cabling to solar arrays. Some of the desired characteristics of these materials include the ability to resist damage from low-Earth-orbit environments (including atomic oxygen and micrometeoroids); insulating materials with high dielectric strengths and flexible enough to prevent damage; and conductors and coatings that have low electrical resistance. A particularly important concern is the need for electrical discharge/arc prevention and suppression if the electrical insulation is breached. In the case of discharges in a vacuum, the insulation material often becomes the gas source to feed the discharge. We have performed some preliminary work to identify, develop, and test these advanced coatings. The approach we are proposing for suppressing the discharge is including in the coating an encapsulated or entrapped electronegative gas, liquid, or solid that are released upon impact or at the onset of a discharge suppressing the growth and continuation of the discharge. This paper discusses the mechanisms for arc creation, preliminary tests we have preformed to suppress arc creation, and a path forward for development of such coatings.
\end{abstract}

\section{Nomenclature}

$\begin{array}{ll}d_{p} & =\text { impact particle diameter }(\mathrm{m}) \\ E & =\text { electric field strength }(\mathrm{V} / \mathrm{m}) \\ m & =\text { micrometeoroid mass }(\mathrm{g}) \\ n_{n} & =\text { neutral density }\left(\mathrm{m}^{-3}\right) \\ n_{\mathrm{tot}} & =\text { atoms of ejected material } \\ P & =\text { pressure }(\mathrm{Pa}) \\ p & =\text { penetration depth }(\mathrm{m}) \\ Q & =\text { charge produced }(\mathrm{C}) \\ t & =\text { time }(\mathrm{s}) \\ v & =\text { impact velocity }(\mathrm{km} / \mathrm{s}) \\ v_{\mathrm{ej}} & =\text { ejecta velocity }(\mathrm{km} / \mathrm{s}) \\ \lambda_{\mathrm{mfp}} & =\text { ionization mean free path }(\mathrm{m}) \\ \sigma & =\text { collision cross-section }\left(\mathrm{m}^{2}\right) \\ \sigma_{p} / \sigma_{t} & =\text { ratio of particle to target tensile strength } \\ \rho_{p} / \rho_{t} & =\text { ratio of particle to target density }\end{array}$

\footnotetext{
* Associate Professor, Engineering Design, Electrical Engineering, and Aerospace Engineering, 213N Hammond Bldg., AIAA Senior Member.

${ }^{\dagger}$ Professor, Electrical Engineering and Space Science, 1301 Beal Ave, AIAA Associate Fellow.
} 


\section{Introduction}

$\mathrm{T}$ here is a need for advanced insulation materials to support systems such as the Momentum Exchange/ Electrodynamic Reboost (MXER) Tether system, other proposed electrodynamic tether missions, and general high voltage applications requiring direct exposure to vacuum/space plasma conditions, such as cabling to solar arrays. Some of the desired characteristics include the ability to resist damage from low-Earth-orbit environments (including atomic oxygen and micrometeoroids); insulating materials with high dielectric strengths and flexible enough to prevent damage; and conductors and coatings that have low electrical resistance. A particularly important concern with present high-voltage tether materials is the need for electrical discharge/arc prevention and suppression if the electrical insulation is breached. For tethers, this breaching can occur due to micrometeoroid or orbital debris impact or simple electrical insulation breakdown due to defects in the insulation. This concern also applies to other high voltage systems, including solar arrays, electric thruster components, and various scientific instruments exposed to vacuum condition. In the case of discharges in a vacuum, the insulation material often becomes the source feeding the discharge.

We have performed some preliminary work to identify, develop, and test advanced coatings for electrodynamic tethers and other high voltage cables that can help prevent and even suppress electrical discharge generation should the coating be breached. The approach we are proposing for suppressing the discharge is by including in the coating an encapsulated or entrapped electronegative gas, liquid, or solid (including nanoparticles) that are released upon impact or at the onset of a discharge suppressing the growth and continuation of the discharge.

This paper discusses the mechanisms for arc creation, preliminary tests we have preformed to suppress arc creation, and a path forward for development of such coatings.

\section{Background}

Electrodynamic tethers (EDTs) are generally considered as a "high-risk, high-payoff" propulsion technology. To date, several orbital EDT and non-conducting tether missions have flown, including TSS-1 and $-1 \mathrm{R},{ }^{\ddagger}$ SEDS- -1 and $-2,{ }^{\S} \mathrm{PMG}^{* *}{ }^{*}$ and TiPS. ${ }^{\dagger \dagger}$ In addition, NASA developed — but did not fly—-the ProSEDS ${ }^{\sharp 1}$ EDT mission. In order to realize the potential of EDTs and hybrid momentum-transfer/electrodynamic tethers, such as the proposed MXER ${ }^{\$ \S}$ system, advanced tether materials must be developed. ${ }^{1}$ These tether materials must be able to survive for long time periods within the harsh low-Earth orbit (LEO) space environment.

One of the largest concerns for tether survivability is the probability of the tether being severed by micrometeoroids and/or orbital debris (MM/OD). It is easy to understand how the impact of these particles with the tether could slice through and/or weaken the tether. Methods to provide redundancy without increasing drag have been identified. However, recent experiments and an examination of the literature reveal another mechanism for significant damage, even if the tether material remains intact after colliding with a particle. This mechanism is arcing caused by the ablation of material from the surface.

The TSS-1R space shuttle mission had an unplanned tether separation that was attributed to a high-voltage failure in the tether insulation that first resulted in a nearly 1-amp arc from the tether conductors to the deployer control boxes inside the deployer system under the multi-layer insulation blankets as the failure point came off the tether reel. ${ }^{2}$ Once the tether moved outside of the deployer in the shuttle payload bay, the arcing event intensified by more than $20 \%$ as the discharge connected directly with the surrounding ionospheric plasma. In Ref. 3, a series of large-chamber experimental simulations was performed to show that trapped gas from voids around the 10 \#34AWG copper wires of the tether would be adequate to allow a discharge to be established and support the discharge current levels to the surrounding ionosphere and agreed with the duration of the discharge. In Ref. 4, Wilbur and Burtner also were able to experimentally demonstrate that the Teflon insulation in the tether could also be a source to feed the discharge plasma. Note that it took almost two minutes from initial arc until the tether actually broke, indicating that if the arc could be suppressed, then the tether's structural integrity might have been maintained.

The ProSEDS tether design and those proposed for follow-on EDT missions have evolved in design such that no trapped gas is contained within the insulation. This is accomplished by applying the insulating coating directly to the conductor, which is aluminum in the case of ProSEDS. ${ }^{5}$ Testing work for the ProSEDS mission, however, identified

\footnotetext{
TSS-1: Tethered Satellite System; TSS-1R: Reflight of TSS

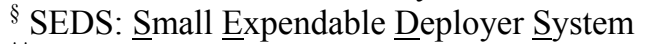

*** PMG: $\underline{\text { Plasma }}$ Motor Generator

†े TiPS: Tether Physics and $\underline{\text { Survivability Experiment }}$

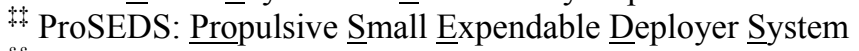

$\$ \S$ MXER: Momentum Exchange, Electrodynamic Reboost, pronounced "mixer"
} 
the possibility of arcing should the tether be impacted by a micrometeoroid while in an open-circuit configuration. The arcing mechanism in this case is due largely to the triple-point created by the insulation, the exposed conductor at high voltage, and the surrounding plasma medium. To mitigate against these possible arcing events, ProSEDS planned to limit the amount of time it would be in the open-circuit configuration. On longer duration EDT missions and for operational MXER-type systems, this is not a viable mitigation methodology.

\section{A. Arcing Mechanisms}

There are three possible mechanisms for developing an arc due to or resulting from the collision of a micrometeoroid or debris particle with a surface (Figure 1). The first is the generation of a plasma cloud due to the energy released in the collision when the surface is also at a high voltage. A second mechanism is ionization of the material ejected as neutral gas. These first two mechanisms would occur for both insulated and bare (i.e., conducting) surfaces. In the insulated case, however, once the underlying conductor is exposed, a third mechanism is possible. This is the development of an arc due to the triple point created by the insulation, the exposed conductor at high voltage, and the surrounding plasma medium. We will examine these processes in more detail below for a large incident particle, which would generally represent a worst case

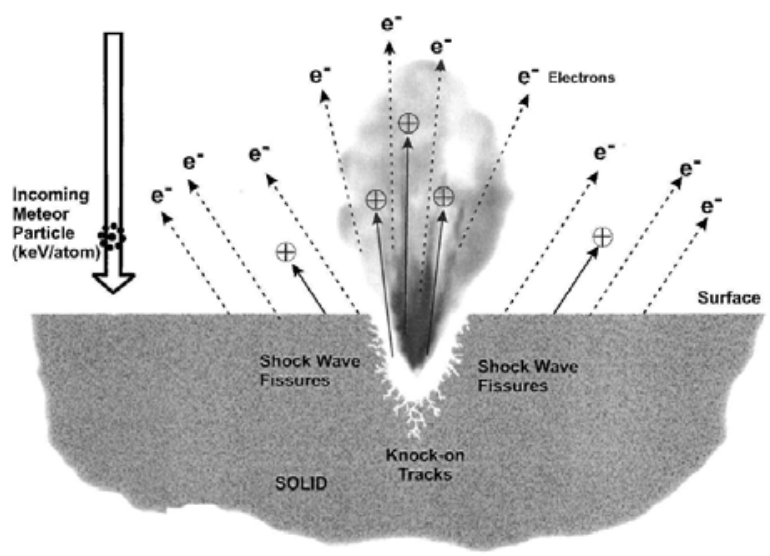

Figure 1. Plasma and neutral gas generated by the hypervelocity impact of a particle on a solid surface [from Ref. 6]. scenario.

As mentioned, when a micrometeoroid collides with a surface, there is enough energy released to generate a plasma cloud at the surface. In Ref. 7 are reported extensive laboratory experiments that were performed to determine the charge, $Q$, produced by this collision and developed the following empirical formula

$$
Q=0.1 m\left(\frac{m}{10^{-11}}\right)^{0.02}\left(\frac{v}{5}\right)^{3.48}
$$

where $Q$ is in coulombs, $m$ is the micrometeoroid mass in grams, and $v$ is its impact velocity in $\mathrm{km} / \mathrm{s}$. Micrometeroids of significant probability to impact the surface ${ }^{* * *}$ can have velocities of up to $\sim 70 \mathrm{~km} / \mathrm{s}$ (e.g., for the Leonids; orbital debris is $\sim 15 \mathrm{~km} / \mathrm{s}$ ) and sizes on the order of $10^{-5} \mathrm{~g},{ }^{8}$ yielding a charge production of $\sim 0.01 \mathrm{C}$. The net charge production is zero, but the plasma has this quantity of charge carriers and due to the different mobilities of ions and electrons, can result in a separation of these charge carriers. ${ }^{9}$

In addition to the plasma cloud generated, the neutral gas generated from the material vaporized during impact may also represent a source for plasma generation provided the electric field over pressure ratio, $E / P$, criterion is met for ionization of the gas. If one assumes the worst case of all material in the impact crater and the incident particle being vaporized ${ }^{\dagger \dagger 1},{ }_{10}^{10}$ then we can calculate pressure as a function of time in the expanding cloud. There are a number of models for crater size found in the literature [cf., Refs. 8 and 11], but a reference model is the "1992C" model given in Ref. 13. The penetration depth, $p$, in meters for the particle is given by the empirical relationship

$$
p=0.7658 d_{p}^{1.056}\left(\frac{\rho_{p}}{\rho_{t}}\right)^{0.476}\left(\frac{\sigma_{p}}{\sigma_{t}}\right)^{0.134} v^{0.806},
$$

where $d_{p}$ is the impact particle diameter in meters, $\rho_{p} / \rho_{t}$ is the ratio of particle to target densities, and $\sigma_{p} / \sigma_{t}$ is the ratio of particle to target tensile strengths. Hence, the model is dependent on the material of both the particle and what it impacts, but for the case of target metals (as in conducting EDTs), the worst case would make these ratios unitary. In this case, for the Leonid micrometeoroid particle mentioned above where $d_{p} \sim 0.1 \mathrm{~mm}$, then $p \sim 1 \mathrm{~mm}$, if

\footnotetext{
*** This represents a "large" particle, although larger particles certainly are available at much lower probabilities.

${ }^{\dagger \dagger}$ Below some velocity threshold, the ejecta are not vaporized and may be considered more as a debris cloud. ${ }^{10,12}$
} 
one assumes that this depth represents the diameter of a half sphere for the crater, then the amount of ejected material may represent some $n_{\text {tot }} \sim 1 \times 10^{19}$ atoms in the case of Al.

Models indicate that the ejecta propagate outward at a few 10's of $\mathrm{km} / \mathrm{s} .{ }^{14} \mathrm{We}$ can assume that the material and particle are vaporized almost instantaneously since the time is takes to travel $1 \mathrm{~mm}$ at $70 \mathrm{~km} / \mathrm{s}$ is on the order of 10 's of nanoseconds. If we assume the vaporized material propagates outward into a solid-angle half-space, density may be thought of as uniform in an expanding half sphere with the radius expanding at the ejecta velocity. This gives a density estimate for the neutrals as a function of time

$$
n_{n}(t)=\frac{n_{\mathrm{tot}}}{\frac{2}{3} \pi\left(v_{\mathrm{ej}} t\right)^{3}}
$$

where $v_{\mathrm{ej}}$ is the ejecta velocity. From this, one can further calculate an ionization mean free path, $\lambda_{\mathrm{mfp}}=1 /\left(n_{n} \sigma\right)$, where $\sigma$ is the collision cross-section. For Al, $\sigma \sim 10^{-19} \mathrm{~m}^{2},{ }^{15}$ which means $\lambda_{\text {mfp }}(t) \sim 2.1 \times 10^{-7} t^{1 / 3}(\mathrm{~m})$. This shows that the initial density will be quite high and the mean free path very small, meaning the ejecta particles likely will not acquire enough energy to ionize $e^{\S \S}$ in the central part of the of the ejecta plume before colliding with other particles for most anticipated tether potentials. However, at the edge of the plume, there will be the correct $E / P$ condition, and in this location an arc may commence. The density in the core plume also decreases as a function of time, also possibly providing the appropriate $E / P$ ratio needed for breakdown. Additionally, smaller particles or those with lower densities do not kick off so much ejecta or may only kick off the coating. This means that the initial density begins much lower, so Townsend ionization in the ejecta plume may be a mechanism for breakdown.

It should be noted that this mechanism for discharge may at times be desirable and has been proposed to discharge spacecraft that have acquired large negative potentials [cf., Ref. 16]. The primary difference between a charged spacecraft and an EDT system is the fact that an EDT system can continue to flow significant amounts of current to maintain the arc or discharge provided the plasma medium can support it, whereas a charged spacecraft will quickly discharge and hence the ionization potential is removed.

The third mechanism for causing an arc resulting from particle impact is the triple-point configuration developed once the particle has impacted the surface and exposed the underlying conductor. The resulting conductordielectric-plasma junction can develop arcs, snapovers, or glow discharges. ${ }^{17}$ There has been considerable research on arcing and snapover with particular application to solar cell arrays [cf., Refs. 18 and 19], in addition to research on arcing on anodized and other insulated surfaces [e.g., Refs. 20 and 21]. In most cases, the arcing process removes more insulating material to help feed the arc. Below, we cover the arcing mechanism in more detail.

In addition to the particle-induced arcing mechanisms explained above, there is also the possibility for arc initiation due to dielectric breakdown, sometimes referred to as snapover. In this process, there is a negatively biased conductor that is covered by a dielectric layer and surrounded by the ambient plasma. Once begun, the arc tends to collect electrons on the dielectric surface and focus ions back to the metal surface or the conductive arc surface ${ }^{22}$ (Figure 2). One of the critical parameters of the arc initiated via dielectric breakdown is the capacitance across the insulation, and hence the stored charge on this capacitor. Another is the ability of the plasma to sustain the current levels required to keep the arc going. It is clear, however, that material ablation from the area surrounding the arc location may provide the necessary medium for plasma generation to support arcs of some considerable magnitude.

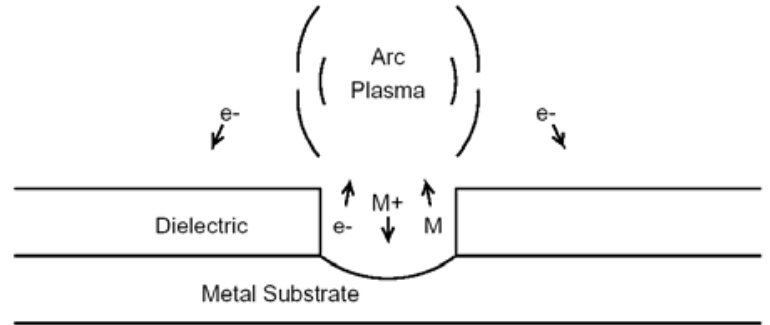

Figure 2. Proposed arc evolution mechanism [from Snyder, 1995].

Although an unbreached insulated conductor submersed in a plasma can have a relatively high voltage applied to it, the data presented in Figure 3 indicate that there is an effective voltage limit between bare (or breached) conductors that is $<1000 \mathrm{~V}$ for almost all near space conditions. Specifically, in the orbits of most LEO satellites and proposed EDT systems (i.e., 200-500 km), the limit appears to be on the order of $300 \mathrm{~V}$ or less. Hence, once a breach occurs, it is important that any continual arcing that eats away at the insulation be stifled.

Models show that this is not exactly the case [cf., refs. 9, 12, and 14], it has more of a $\cos \theta$ distribution. Nevertheless, this approximation will suffice here.

$\S \S \S \mathrm{Al}$ has an ionization potential of $\sim 6 \mathrm{eV}$, and most materials fall in the $5-15 \mathrm{eV}$ range. 


\section{Proposed Solution}

As a method for mitigating the arcing concerns caused by the mechanisms listed above, we propose the entrapment of an arc suppressing gas and/or material within the coating that, when impacted, sputtered, or sublimated away, releases simultaneously with the coating and/or underlying conductor material. Our initial candidate for this material, chosen because of its many desirable properties, is sulfur hexafluoride. In this section, we first discuss the properties of sulfur hexafluoride that made it an excellent candidate for the feasibility studies we conducted (we will later discuss a number of other candidate materials that were uncovered during our research, which should be tested in the future for their arc-suppressing properties). We then look at the preferred entrapment method, which is microencapsulation. The microspheres, first filled with $\mathrm{SF}_{6}$, would then be mixed with an "off-the-shelf" coating (such as TOR ${ }^{\mathrm{TM}}$ polymer solution made by Triton Systems) or in theory any new coatings developed as part of other efforts.

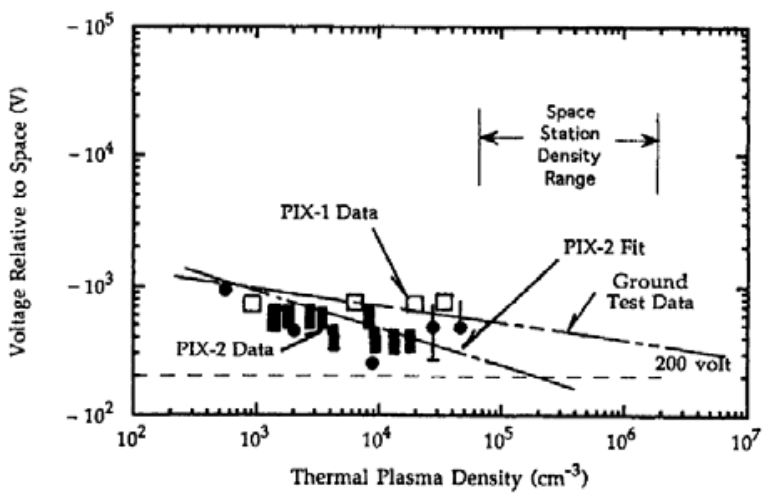

Figure 3. Voltage breakdown as a function of local plasma density [from Ref. 23].

\section{Sulfur Hexafluoride}

Sulfur hexafluoride $\left(\mathrm{SF}_{6}\right)$ is an extremely efficient insulator widely used in the electrical transmission industry and in other high voltage systems. Properties that make it a good insulator include a high single-ionization energy and its ability to inhibit electrical discharges due to its large cross section for electron capture, i.e., it is highly electronegative. In addition, should it be ionized, $\mathrm{SF}_{6}^{+}$is not a stable ion and neither are its higher ionization states. ${ }^{24}$

Arcing in $\mathrm{SF}_{6}$, in general, results in no or minimal lessening of the dielectric strength of the gas, although in the presence of other gases, several decomposition by-products are formed. The resulting mixture, however, is not degraded in dielectric strength. ${ }^{25}$ Because $\mathrm{SF}_{6}$ tends to trap free electrons, runaway generation of electrons needed to form a conducting path to sustain an arc is difficult. At temperatures above $5000 \mathrm{~K}$ as could easily be seen in an arc, $\mathrm{SF}_{6}$ is completely decomposed into atomic ions and electrons $\left(\mathrm{e}^{-}, \mathrm{F}^{+}, \mathrm{S}^{+}, \mathrm{S}^{++}\right.$, etc.), but as the temperature drops $(<3000 \mathrm{~K})$ moving away from the arc area, molecular fragments $\left(\mathrm{SF}_{4}, \mathrm{SF}_{2}, \mathrm{~F}_{2}, \mathrm{~S}\right.$, and $\left.\mathrm{F}\right)$ are quickly found. ${ }^{26}$

$\mathrm{SF}_{6}$ is also chemically inert and not toxic because fluorine is a strong oxidizer and no other element will substitute in preference to fluorine in $\mathrm{SF}_{6}{ }^{25}$ Hence, $\mathrm{SF}_{6}$ should not react with the material used in the tether coating or with the microsphere encapsulant. Ref. 27 provides data on the total electron attachment cross section, $\sigma_{a, t}(\varepsilon)$,

for $\mathrm{SF}_{6}$, from which we can determine the amount of $\mathrm{SF}_{6}$ necessary to quench or keep an arc from occurring. Determination of exact quantities of gas needed was one of the tasks undertaken as part of our initial investigations and are presented later.

As mentioned, the method we have settled on for entrapment of the electronegative gas, in our case $\mathrm{SF}_{6}$, is in a microsphere, an example of which is shown in Figure 4. Discussions with microencapsulation companies indicate that the microspheres can be manufactured in sizes ranging from on the order of $100 \mathrm{~nm}$ up through a few mm depending on the process chosen. The wall thickness can be such that it occupies anywhere from $5 \%$ to $50 \%$ of the volume of the microsphere. The wall material also depends on the process, but can be a polymeric material and is generally something that may be specified, provided it is compatible with the process and the material to be encapsulated. This will allow flexibility in selecting the encapsulant to ensure that it is also compatible with the base insulation.

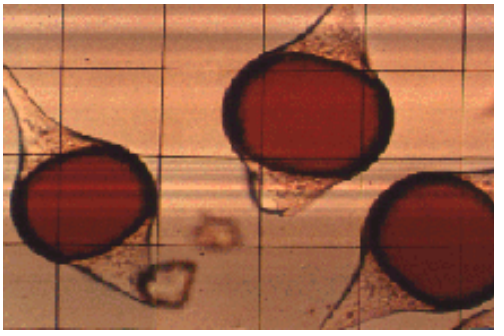

Figure 4. Microencapsulated materials (grid spacing $1 \mathrm{~mm}$ ).

\section{Proof-of-Concept Results}

The goal of proof-of-concept testing was to show that the presence of an electronegative gas - in a cavity within an insulated conductor biased to a high voltage and placed in a vacuum environment-would reduce the prevalence or, ideally, eliminate any arcing. To this end, proof-of-concept testing involved designing and fabricating an experimental test apparatus; obtaining $\mathrm{SF}_{6}$ and determining how to fill the cavities; and performing a series of 
experiments in ambient air and a vacuum chamber. Our tests successfully demonstrated the efficacy of entrapped $\mathrm{SF}_{6}$ as an arc suppressant.

\section{A. Test Apparatus Fabrication}

One of the most critical issues to resolve in performing these tests was to find a way that a small quantity of gas could be trapped in a reservoir that subsequently could be punctured. We fabricated and evaluated several different designs before converging on the experimental setup described here, which was used for these feasibility experiments. Each reservoir was constructed of a Swagelok SS-42S4G on-off ball valve (Figure 5a), to one side of which was connected a lecture flask of $\mathrm{SF}_{6}$ for filling purposes, and to the other side was a blind rivet prepared in the manner described below and shown in Figure 5b. When the ball valve is open, the reservoir can be filled and then closed off by closing the ball valve. The rivet insert occupies almost all extra volume (seen in the transparent view of the ball valve in Figure 5a) and allows the size of the reservoir to be set exactly. Insulation [0.500in. $(1.27-\mathrm{cm})$ diameter Kapton tape disks with total tape thickness of $0.0025 \mathrm{in}$. $(0.064 \mathrm{~mm})$ : film is 0.001 in. $(0.025 \mathrm{~mm})$ and silicone adhesive is 0.0015 in. $(0.038 \mathrm{~mm})$ ] is then placed over the reservoir. Once filling is complete (the filling process is described below), the gas bottle is removed so that the assembly can be placed in a vacuum chamber.

To create the cavity, a hole was drilled

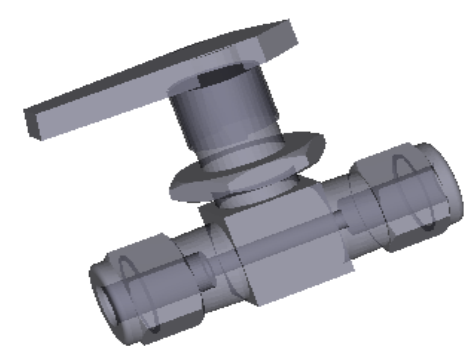

(a)

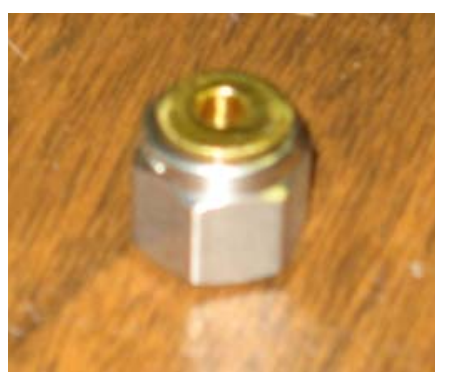

(b)

Figure 5 (a) Swagelok ball valve showing internal plumbing. (b) Drilled out blind rivet inside a Swagelok fitting that connects to one end of the ball valve.

down the center of a blind rivet, which was then cut off to a length of $1.5 \mathrm{~cm}$. This rivet was then swaged onto the fitting on the ball valve. The blind rivet ensured that any extraneous volume of the Swagelok valve was filled, leaving only a small volume drilled out for the addition of $\mathrm{SF}_{6}$. After the rivet was machined, it was cleaned thoroughly with ethyl alcohol to remove any contaminants that might have been left over from the machining process. This, and the filling procedure, ensured as pure a sample of $\mathrm{SF}_{6}$ as possible. Once the cleaning was complete, the rivet was fitted in the valve, and the valve assembly was constructed.

The valve assembly (Figure 6) was constructed in such a way as to isolate the solenoids from the high voltage valves. The valves were affixed to a $1 / 8^{\prime \prime}(3.2 \mathrm{~mm})$ thick piece of steel, which was bolted to a Plexiglas base. The Plexiglas base was used to isolate the grounded solenoids from the valves, which were at a high voltage. As for the solenoids, they were bolted to another $1 / 8^{\prime \prime}$ thick piece of steel, located above the valves, and aligned such that the plunger would puncture the Kapton tape in the center of the cavity. The five solenoids were wired in such a way as to allow each one to be fired independently of the others. No

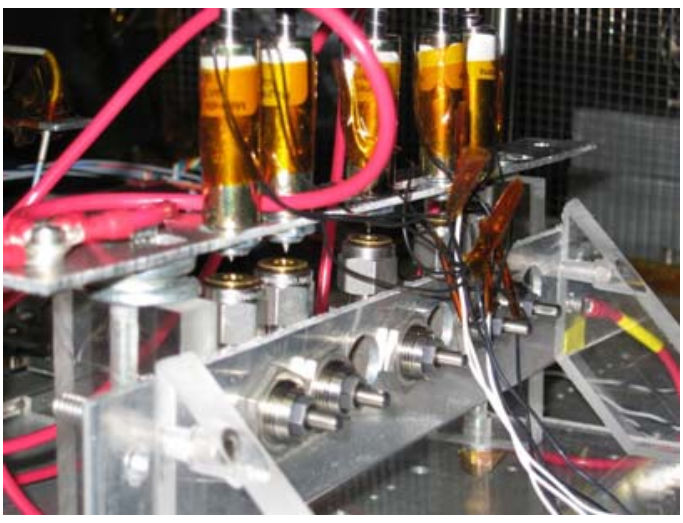
Figure 6 Picture of cavities located immediately under solenoid plungers and the assembly placed in the vacuum chamber.

Kapton tape insulation was placed on the cavity that was left open to the ambient atmosphere. For the air-filled cavity, Kapton tape was simply placed over the cavity prior to placing the valve assembly into the chamber. Three cavities were then filled with $\mathrm{SF}_{6}$ at $1 \mathrm{~atm}$, providing a total of five cavities for each test run.

The procedure for filling the cavities containing the $\mathrm{SF}_{6}$ is as follows. First a piece of Kapton tape was loosely placed over the cavity, then the $\mathrm{SF}_{6}$ was turned on and allowed to flow for a brief period of time to ensure that all the air was expelled from the cavity, and a pure sample of $\mathrm{SF}_{6}$ was sealed with the Kapton Tape. Once the air was expelled from the cavity, the Kapton tape sealed the cavity and the valve was closed. This was done in such a manner as to ensure that no bubble of gas formed above the cavity. There were two reasons for this, one being that if a bubble of gas formed above the cavity, there was a greater chance for the $\mathrm{SF}_{6}$ to break the seal of the tape once a vacuum was reached. The second reason was that this would increase the volume of gas present.

Once the gas was sealed inside the cavities, the valve assembly was placed inside a vacuum chamber, and the high voltage lines were connected. See Figure 7 for a wiring diagram of the assembly, the red lines are high voltage, the blue lines are lower voltage, and the black lines carry the current to allow the solenoids to fire. Once everything 
was properly connected, the chamber was sealed, and the chamber was pumped down to pressure. The operating pressure depended on the specific experiment.

\section{Experimental Procedure}

The experiments were performed in the following manner. First, solenoid one was fired to determine if the ambient pressure was enough to support an arc. To determine if an arc was present, an oscilloscope was set up to measure the voltage between the solenoid plungers and ground across a resistor. After solenoid one was fired, solenoid two was fired. Solenoid two

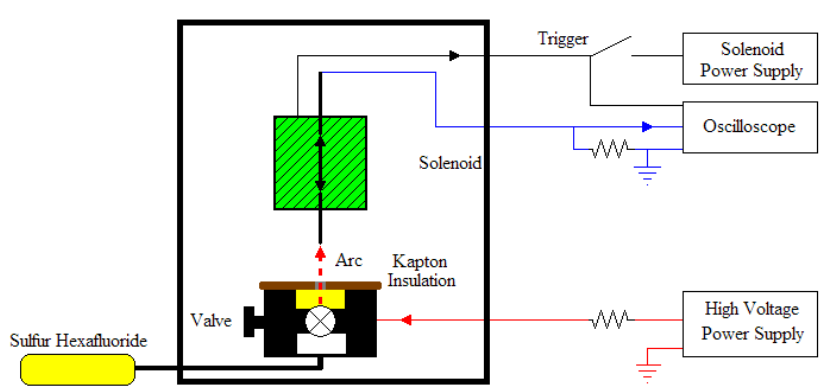

Figure 7. Wiring diagram for high voltage experiments in chamber. Note, $\mathrm{SF}_{6}$ bottle was disconnected before running experiments. acted as a control, to make sure that the valve assembly was working. Once the first two solenoids were fired, the three solenoids with the $\mathrm{SF}_{6}$ were fired.

\section{Experimental Results}

Four different experiment runs were performed using the valve assembly described above. In the first run, solenoid 1 (which was left open to the ambient pressure), was fired with the vacuum chamber at a pressure of $0.64 \times 10^{-3}$ Torr, and a potential difference between the valves and the solenoids of up to $5000 \mathrm{~V}$, with no arc present. To determine if the assembly was working properly, solenoid 2 was fired, which was full of air at standard temperature and pressure (STP). An arc formed as was expected since the air in the cavity rapidly ionized facilitating an arc.

Solenoid 3, which was filled with $\mathrm{SF}_{6}$, was then fired and no arc was generated. We decided to hold off firing solenoids 4 and 5 until the pressure in the chamber was higher. This was decided because no arc was generated with solenoid 1, which was left open to atmosphere, and this would make it difficult to determine if there was no arc due to the $\mathrm{SF}_{6}$ or because there was not enough background pressure. Hence, the chamber was left overnight and the last two solenoids were fired the next morning. By this time, the pressure in the chamber was about $10^{-2}$ Torr, and an arc was generated with solenoid 1. Solenoid 4, which was filled with $\mathrm{SF}_{6}$ was then fired, and it was determined that no arc occurred.

Solenoid 4 was fired again shortly after the first test, and this time an arc occurred. This indicates that the $\mathrm{SF}_{6}$ originally had suppressed the arc, but once the gas discharged into the chamber, the ambient pressure was sufficient to cause an arc. This same result was repeated when solenoid 5 was fired immediately after the results were obtained for solenoid 4. The potential difference between the valve assembly and the solenoid plunger for this test was approximately 1.135 $\mathrm{kV}$.

A second set of experiments was performed with the pressure in the chamber at $4.8 \times 10^{-3}$ Torr, and the potential difference was $1500 \mathrm{~V}$. During this test, the only solenoid to generate an arc was solenoid 3 . The waveform is shown in Figure 8 , note the small change in the high voltage return signal was due to the range settings of the oscilloscope. It is believed that the arcing was not actually being measured in between the solenoid and the valve, but actually background arcing elsewhere in the chamber, as no visible arc was seen

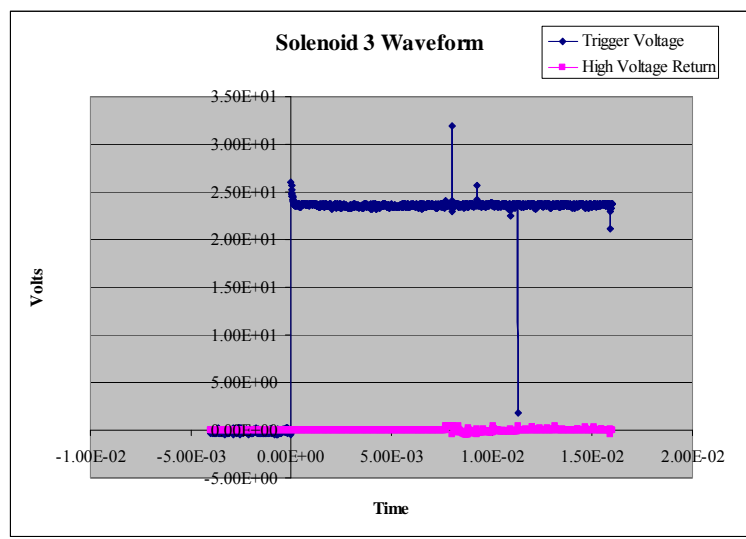

Figure 8. Waveform of solenoid 3 showing arcing present (pink line).

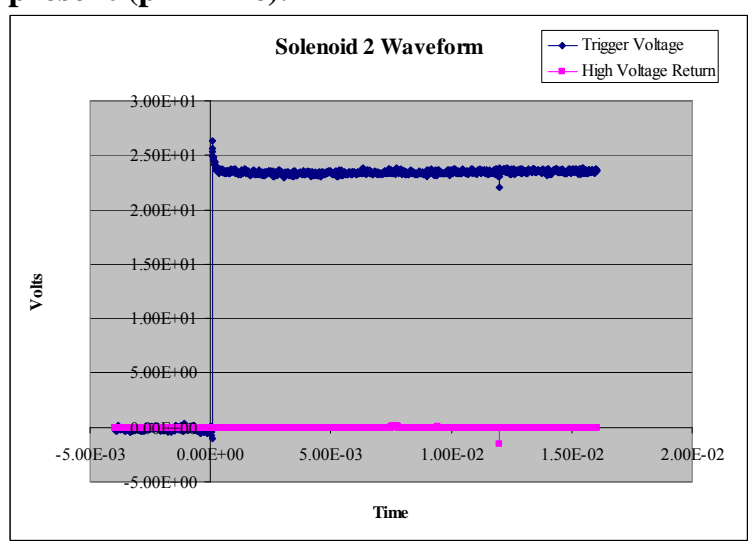

Figure 9. Waveform of solenoid 2 with $3.05-\mathrm{mm}$ diam. cavity showing arcing present (pink line) (experiment 4). between the solenoid and the valve. 
To verify this result, this set of experiments was repeated. During this second test, the ambient pressure was $4.7 \times 10^{-3}$ Torr, and the applied voltage was $1500 \mathrm{~V}$. Solenoid 1 did not arc, solenoid 2 did, as well as solenoids 3 and 4. Again, when solenoid 2 arced, which was filled with air, a visible spark between the plunger and the valve was seen. This was in contrast to the firing of solenoids 3 and 4 . When solenoids 3 and 4 fired, no arc was visible between the solenoid and the valve; however, there was background glow and arcing taking place elsewhere in the chamber. It is believed that the background arcing is what the oscilloscope was reading. Solenoid 5 did not arc; however, there appeared to be a bubble in the tape of solenoid five after the chamber had been pumped down, and then it appeared that the gas had leaked out.

The first three sets of experiments were conducted with a cavity size of approximately $5.16 \mathrm{~mm}$ in diameter (cavity volume $314 \mu \mathrm{L}$ ). A fourth set of experiments was performed in the vacuum chamber with a cavity diameter of approximately $3.05 \mathrm{~mm}$ (cavity size $100 \mu \mathrm{L}$ ). During this experiment, the ambient pressure was $4.6 \times 10^{-3}$ Torr, and the applied voltage was $1400 \mathrm{~V}$. Again no arc was generated from solenoid 1, which again was left open to ambient; however, solenoid 2, which was filled with air, and solenoids 3 and 4 (both full of $\mathrm{SF}_{6}$ ) arced. The arc generated by solenoid 2, was larger and occurred earlier than the arcs of both solenoids 3 and 4 . Figure 9 shows the waveform for solenoid 2 during this experiment, and Figure 10 shows the waveform for solenoid three.

Numerous experiments were also performed with the 3.05-mm-diam. cavity, with the entire valve assembly at STP. Figure 11 provides is a figure of solenoid one, which was left open to the ambient atmosphere, in this case, STP, with an applied voltage of $4000 \mathrm{~V}$. A very large arc was present; however, when a cavity with $\mathrm{SF}_{6}$ was tested, no arc was present.

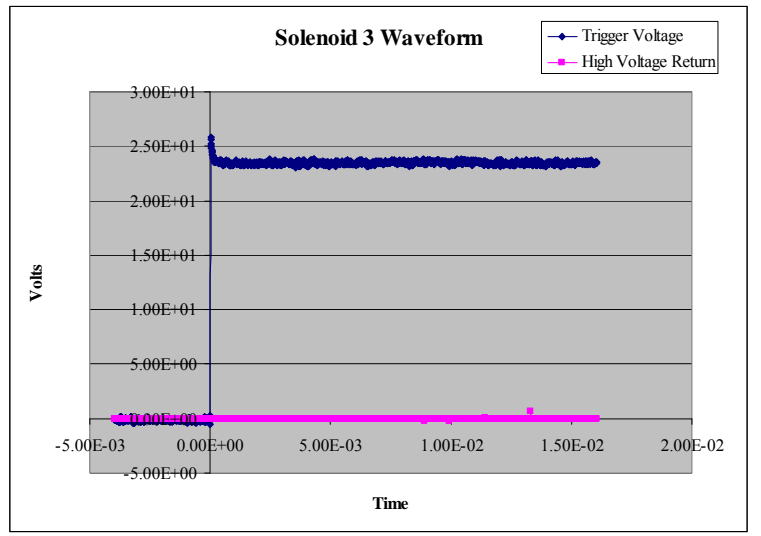

Figure 10. Waveform of solenoid 2 with 3.05mm-diam. cavity showing reduced arcing present (pink line) (experiment 4).

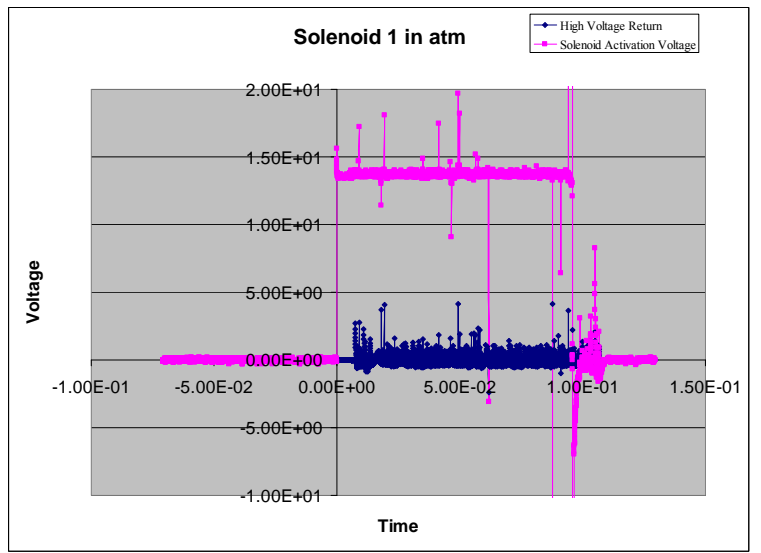

Figure 11. Solenoid one fired at STP, and left open to the ambient atmosphere showing heavy arcing present (pink line)

\section{Discussion of Results}

Our proof-of-concept results agree with the original assumptions and provide sufficient evidence of success to support continued research into this entrapped materials for arc suppression. Specifically, these experiments indicate that:

- The presence of an electronegative material, in this case $\mathrm{SF}_{6}$ gas, nearby a high voltage conductor with breached insulation can mitigate an initial arc.

- Arc mitigation depends on the quantity of the suppressant available and, even when available only in quantities that do not eliminate the arc, it reduces the severity of the arc event and increases the time before arc onset.

If we assume that the vaporized insulation material contains on the order of $1 \mu \mathrm{L}$ of microencapsulated $\mathrm{SF}_{6}$, then we can calculate how many molecules this contains. 1 mole of gas at standard temperature and pressure (STP) occupies a volume of $22.4 \mathrm{~L}$. (A mole of $\mathrm{SF}_{6}$ has a mass of $146.1 \mathrm{~g}$ ). Given that there are $6.02 \times 10^{23}$ molecules per mole, then $1 \mu \mathrm{L}$ of $\mathrm{SF}_{6}$ contains on the order of $3 \times 10^{16}$ molecules. From Eqn. (1), a very energetic MM/OD collision will create a plasma cloud containing $\sim 0.01 \mathrm{C}$. Since $1 \mathrm{C}=6.24 \times 10^{18}$ charges, then $\sim 6 \times 10^{16}$ charges are created, which is the same order of $\mathrm{SF}_{6}$ molecules released. Hence, these zeroth-order calculations indicate that in coatings of reasonable thickness it will be possible to entrap enough gas to quench most arcing events. 


\section{B. Other Candidate Materials}

We have also attempted to uncover materials other than SF6 as candidates for use as an arc suppressant. $\mathrm{SF}_{6}$ is the arc suppressant with which we have the most experience, and for which the most background data is available. However, nanoparticles offer a very interesting possibility that should be explored as well. Buckminster fullerenes (most prevalent is of form $\mathrm{C}_{60}$ ), also known as "buckyballs", are often described as being in the shape of a soccer ball and are about $0.7 \mathrm{~nm}$ in diameter (4.5 times as large as a hydrogen atom, or a bit less than a billionth of a meter). Its atomic structure imparts unique properties to this material: electrically insulating; highly electronegative; can be polymerized; can be sublimed; and can be dissolved.

Since fullerenes are highly electronegative, they are a candidate arc suppressant in the same manner that $\mathrm{SF}_{6}$ is. In addition, their large mass prevents them from accelerating quickly once they have picked up electrons, reducing the probability that they will ablate additional insulation material before the plasma cloud dissipates below critical density for arcing. The electron attachment cross-section for $\mathrm{C}_{60}$ is on the order of that of $\mathrm{SF}_{6}$. In addition, above $\sim 0.5 \mathrm{eV}$, the probability that a captured electron will form a long-lived $\mathrm{C}_{60}^{-}$anion is about $50 \% .{ }^{28}$ Another property of fullerenes that might make it ideal for this application is that they are highly resistant to fragmentation even at high impact velocities ${ }^{29}$ making them likely to survive MM/OD impacts. Many of the fullerene forms $\left(\mathrm{C}_{60}\right.$ to $\mathrm{C}_{70}$ and higher) share similar properties; thus, obtaining a high purity form of $\mathrm{C}_{60}$ should not be necessary, which should significantly lower the cost of the material.

Other electron absorbing/adhering nanoparticles, even such simple elements as copper or nickel, could have the same effect, potentially allowing a wide variety of nanomaterials to have suppressant effects. Variable conductivity silicon nanoparticles could also be examined. These have the significant benefit over $\mathrm{SF}_{6}$ of being solid phase at room temperature, allowing the addition of high densities of suppressant without additional difficulty.

\section{Future Directions of Research}

Future research should be focused on identifying the best materials to provide arc suppression, and then amongst the best, determine which material is most compatible with integration into existing tether insulation technology to provide a viable product. Using the data acquired with $\mathrm{SF}_{6}$ during our proof-of-concept testing as a baseline, the performance of all materials identified will then be compared and the optimal arc-suppressants selected. These data also will serve as a baseline for later experiments when the materials are mixed with tether insulation, in order to verify that there are no interactions between the arc suppressant and the other materials in the tether coating that adversely affect the arc-suppression characteristics.

Once suitable candidates have been identified, work will begin on combining these suppressant materials with existing tether insulation materials. The new insulation must retain the necessary structural and chemical properties of the base tether coating, including high tensile strength, ultraviolet light and atomic oxygen resistance, and high voltage standoff. Experimentation and testing will be required to find the appropriate mix, and some compromise between arc suppression capability and other characteristics may be required. Several candidate insulations should be developed and tested. Potential configurations include adding suppressant directly within the chemistry of existing coatings (Figure 12a), developing a separate coating that would be applied as an interior layer (Figure $12 \mathrm{~b}$ ), or mixing in chemically isolated encapsulated pockets of suppressant that are only released in the event of

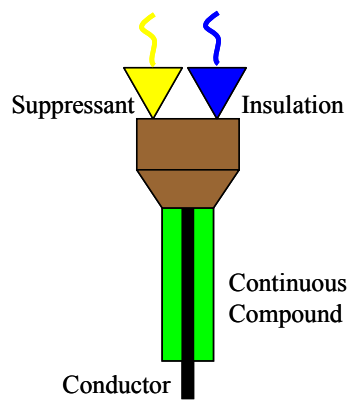

(a)

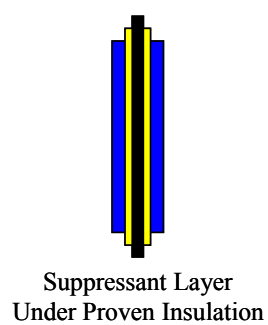

(b)

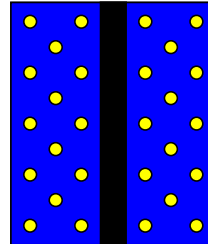

Encapsulated Suppres: Mixed Into Insulatic

(c)

Figure 12. Potential coating configurations insulating material (blue) and suppressant (yellow) include (a) adding suppressant directly within the chemistry of existing coatings-figure shows two materials mixing together to form new material; (b) separate coating that would be applied as an interior layer; (c) mixing in chemically isolated encapsulated pockets of suppressant.

insulation breach (Figure 12c).

The insulation samples thus created must then be tested in a variety of configurations: applied to both flat plate and wire conductors, single and multi-strand, representative of EDTs and other high voltage space/vacuum wiring. In addition to mechanical breach of the insulation material as demonstrated during proof-of-concept tests, 
experimentation might include melting or use of pulsed laser ablation to cause insulation breaches within a vacuum, to more accurately represent the high energy nature of orbital debris impact.

Although this research effort is directed toward the development of an arc-suppression coating for EDTs, the technology would have broader application on spacecraft. There are a number of other surfaces and features found on spacecraft that also carry the risk of arcing. One surface already mentioned is the solar cell array. Although the coating could probably never be made transparent enough to place directly on the cover glass of the solar cells, it could be placed on surfaces between cells. Figure 13 shows how

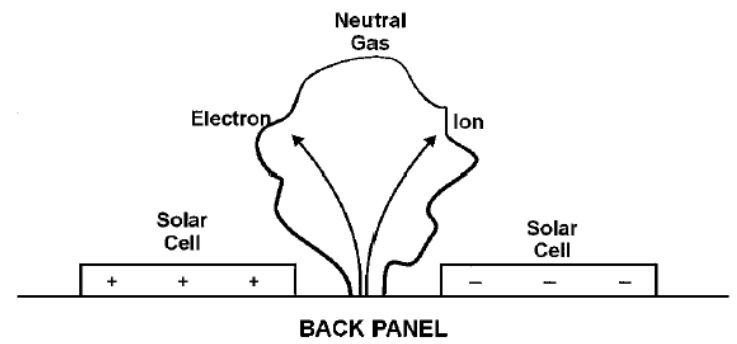

Figure 13. Electrons, ions, and neutral gas generated by a hypervelocity impact on a solar panel [from Ref. 6].

this would be effective for some impacts. If the arc initiates in the region between the solar cells, the ionized gas might cause a shorting path to exist between two solar cells held to different potentials. The effect of a sustained low-level discharge is system degradation and current drain from batteries; an avalanche discharge might cause complete power supply failure. ${ }^{6}$ The new coating proposed here would keep this path from forming by removing ionizing electrons via the electronegative gas released at the same time.

Another feature found on many spacecraft is the exposed cable bundle, which may also be impacted by debris or experience arcing during its lifetime. If proven effective, such cables would benefit from a similar technique of entrapping electronegative material within the insulation.

\section{Summary}

This paper has detailed the results of a proof-of-concept testing effort focused on developing coating solutions to mitigate the effects of arcing. The novel aspect of our proposal is the entrapment of an arc suppressing gas and/or material within the coating that, when impacted, releases simultaneously with the ablated material. Additionally, after the initial impact it will serve to keep arcs from developing due to the triple point by releasing new gas along with the insulation that is feeding the arc. If the source of the electronegative gas is a liquid or solid phase, then a significant quantity of suppressant can be stored in a small volume of insulation

Proof-of-concept testing has shown that the ideas proposed herein have merit as arc-suppressants for EDT insulation. Preliminary experiments indicate that the technique of electronegative material encapsulation is effective at suppressing or reducing arcing in the event of insulation breach. In addition to $\mathrm{SF}_{6}$, research indicates that there are a variety of materials which could be effective in this regard and techniques are available to include these materials in viable EDT coatings.

\section{Acknowledgments}

This work was partially supported by NASA Phase I SBIR grant \#NNM06AA36C through ElectroDynamic Applications (EDA), Inc. with which both Gilchrist and Bilén are affiliated. Gilchrist is part owner at EDA. The authors which to thank D. Morris for his contributions to this project and A. Gross and C. Valentino for their help on the experimental portion of this work.

\section{References}

${ }^{1}$ Johnson, L., Materials Science for Advanced Space Propulsion Workshop, Huntsville, AL, 8-10 October 2001, available at http://msad.msfc.nasa.gov/mwd.

${ }^{2}$ Szalai, K., "TSS-1R Mission Failure Investigation Board: Final Report," NASA, 1996.

3 Gilchrist, B.E., Bonifazi, C., Bilén, S.G., Raitt, W.J., Burke, W.J., Stone, N.H., and Lebreton, J.P. "Enhanced Electrodynamic Tether Currents Due to Electron Emission from a Neutral Gas Discharge: Results from the TSS-1R Mission," Geophysical Research Letters, Vol. 25, No. 4, pp. 437-440, 1998.

${ }^{4}$ Wilbur, P.J., and Burtner, D., "Hollow-Cathode Plasma Contacting Insights Gained from the Electrodynamic Tether Mission," IEPC-99-218, 1999.

${ }^{5}$ Curtis, L., Vaughn, J., Welzyn, K., and Carroll, J., "Development of the Flight Tether for ProSEDS," Proceedings of Space Technology and Applications International Forum - STAIF 2002, Albuquerque, NM, Feb. 3-6, 2002, Melville, NY, American Institute of Physics, pp. 383-392, 2002.

${ }^{6}$ Lai, S.T., Murad, E., and McNeil, W.J., "Hazards of Hypervelocity Impacts on Spacecraft," Journal of Spacecraft and Rockets, Vol. 39, No. 1, pp. 106-113, 2002. 
${ }^{7}$ McBride, N., and McDonnell, J.A.M., "Meteoroid Impacts on Spacecraft: Sporadics, Stream, and the 1999 Leonids," Planetary and Space Science, Vol. 47, pp. 1005-1013, 1999.

${ }^{8}$ McDonnell, J.A.M, "HVI Phenomena: Applications to Space Missions," International Journal of Impact Engineering, Vol. 23, pp. 597-619, 1999.

Crawford, D.A., and Schultz, P.H., "Electromagnetic Properties of Impact-Generated Plasma, Vapor and Debris," International Journal of Impact Engineering, Vol. 23, pp. 169-180, 1999.

10 Ratcliff, P.R., Reber, M., Cole, M.J., Murphy, T.W., and Tsembelis, K., "Velocity Thresholds for Impact Plasma Production," Advances in Space Research, Vol. 20, No. 8, pp. 1471-1476, 1997.

${ }^{11}$ Gardner, D.J., McDonnell, J.A.M., and Collier, I. "Hole Growth Characterization for Hypervelocity Impacts in Thin Targets," International Journal of Impact Engineering, Vol. 19, No. 7, pp. 589-602, 1997.

${ }^{12}$ Schonberg, W.P., "Characterizing Secondary Debris Impact Ejecta," International Journal of Impact Engineering, Vol. 26, pp. 713-724, 2001.

${ }^{13}$ McDonnell, J.A.M., and Sullivan, K., "Hypervelocity Impacts on Space Detectors: Decoding the Projectile Parameters," in Hypervelocity Impacts in Space, J.A.M. McDonnell, editor, University of Kent, pp. 28-36, 1992.

${ }^{14}$ Corvonato, E., Destefanis, R. and Faraud, M., "Integral Model for the Description of the Debris Cloud Structure and Impact," International Journal of Impact Engineering, Vol. 26, pp. 115-128, 2001.

${ }^{15}$ Pinho, G.P., Schittenhelm, H., Duley, W.W., Schlueter, S.A., Jahani, H.R., and Mueller, R.E., "Energy Distributions in the Laser Ablation of Metals and Polymers," Applied Surface Science, Vol. 127-129, pp. 983-987, 1998.

${ }^{16}$ Walker, D.N., Amatucci, W.E., Bowles, J.H., Fernsler, R.F., Siefring, C.L., Antoniades, J.A., and Keskinen, M.J., "Discharging Spacecraft through Neutral Gas Release: Experiment and Theory," Journal of Geophysical Research, Vol. 104, No. A6, pp.12,472-12,485, 1999.

${ }^{17}$ Vayner, B., Galofaro, J., Feguson, D., De Groot, Thomson, W.C., Dennison, J.R., and Davies, R. "The ConductorDielectric Junctions in a Low Density Plasma," NASA Technical Memorandum TM-1999-209408, 1999.

${ }^{18}$ Hastings, D.E., "The Arcing Rate for a High Voltage Solar Array: Theory and Experiments," The Behavior of Systems in the Space Environment, Norwell, MA, Kluwer Academic Publishers. 245: 779-794, 1993.

${ }^{19}$ Upschulte, B.L., Marinelli, W.J., Carleton, K.L., Weyl, G., Aifer, E., and Hastings, D.E., “Arcing on Negatively Biased Solar Cells in a Plasma Environment," Journal of Spacecraft and Rockets, Vol. 31, No. 3, pp. 493-501, 1994.

${ }^{20}$ Carruth, Jr., M. R., Vaughn, J.A., Bechtel, R.T., and Gray, P.A., "Experimental Studies on Spacecraft Arcing," Journal of Spacecraft and Rockets, Vol. 30, No. 3, pp. 324-327, 1993.

${ }^{21}$ Vayner, B.V., Doreswamy, C.V., Ferguson, D.C., Galofaro, J.T., and Snyder, D.B., “Arcing on Aluminum Anodized Plates Immersed in Low-Density Plasmas," Journal of Spacecraft and Rockets, Vol. 35, No. 6, pp. 805-811, 1998.

${ }^{22}$ Snyder, D.B., "Dynamic Interactions between Ionospheric Plasma and Spacecraft," URSI Symposium, Brussels, Belgium, 26-27 April 1995.

${ }^{23}$ Latham, R.V., High Voltage Vacuum Insulation: Basic Concepts and Technological Practice, $2^{\text {nd }}$ edition, Academic Press, 1995.

${ }^{24}$ Griffiths, I.W., Parry, D.E., and Harris, F.M., "Experimental and Theoretical Study of the Double Ionization of the Sulphur Hexafluoride Molecule to Singlet and Triplet Electronic States of Its Dication," International Journal of Mass Spectroscopy, Vol. 185/186/187, pp.651-662, 1999.

${ }^{25}$ Boggs, S.A., "Sulphur Hexafluoride: Introduction to the Material and Dielectric," IEEE Electrical Insulation Magazine, Vol. 5, No. 5, pp. 18-21, 1989.

${ }^{26}$ Boggs, S.A., and Schramm, H.-H., "Current Interruption and Switching in Sulphur Hexafluoride," IEEE Electrical Insulation Magazine, Vol. 6, No. 1, pp. 12-17, 1990.

27 Christophorou, L.G., and Olthoff, J.K., "Electron Attachment Cross Sections and Negative Ion States of SF 6 ," International Journal of Mass Spectroscopy, Vol. 205, pp.27-41, 2001.

${ }^{28}$ Kasperovich, V., Tikhonov, G., and Kresin, V.V., "Low-energy Electron Capture by Free $\mathrm{C}_{60}$ and the Importance of Polarization Interaction," Chemical Physics Letters, Vol. 337, pp. 55-60, 30 March 2001.

${ }^{29}$ Campbell, E.E.B., Fullerene Collision Reactions, Kluwer Academic Publishers, Boston, 208 pp., 2003. 